\title{
Perfil das gestantes com Síndrome Hipertensiva Específica da Gestação atendidas nas Unidades Básicas de Saúde na Cidade de Cáceres-Mato Grosso, Brasil
}

\author{
Profile of pregnant women with Pregnancy-Specific Hypertensive Syndrome attended at Basic \\ Health Units in the City of Cáceres-Mato Grosso, Brazil
}

Perfil de gestantes con Síndrome Hipertensivo Específico del Embarazo atendidas en Unidades

Básicas de Salud de la Ciudad de Cáceres-Mato Grosso, Brasil

Recebido: 03/09/2021 | Revisado: 13/09/2021 | Aceito: 16/09/2021 | Publicado: 17/09/2021

\author{
Maria das Dores dos Santos \\ ORCID: https://orcid.org/0000-0002-3005-9324 \\ Universidade do Estado de Mato Grosso, Brasil \\ E-mail: maria.doracaceres@gmail.com \\ Danyella Rodrigues de Almeida \\ ORCID: https://orcid.org/0000-0003-1181-9321 \\ Universidade do Estado de Mato Grosso, Brasil \\ E-mail: dannypirelli@hotmail.com \\ Maria Lucia de Oliveira \\ ORCID: https://orcid.org/0000-0001-8727-7755 \\ Universidade do Estado de Mato Grosso, Brasil \\ E-mail: marialuciaoliveira33@hotmail.com \\ Stefanny Maria Santana de Campos \\ ORCID: https://orcid.org/0000-0001-8667-3190 \\ Universidade do Estado de Mato Grosso, Brasil \\ E-mail: stefanny.msc@gmail.com
}

\begin{abstract}
Resumo
A maioria das gestações transcorre sem intercorrências, entretanto algumas mulheres, apresentam evolução desfavorável classificadas como gravidez de risco. Entre as doenças que podem ser desenvolvidas no período gestacional destaca-se a Síndrome Hipertensiva Especifica na Gestação (SHEG), considerada uma das principais causas de mortalidade materna fetal. O objetivo desse estudo é conhecer o perfil das gestantes atendidas nas Unidades Básica de Saúde (UBS) na cidade de Cáceres-MT no ano de 2020, visando identificar os fatores de riscos que influenciam no aparecimento da doença e suas complicações. Trata-se de um estudo de campo de caráter descritivo quali-quantitativo, realizado com 16 gestantes entre os meses de junho a julho de 2020. Para a coleta de dados foi elaborado e aplicado um questionário com perguntas fechadas e abertas. Houve maior prevalência de mulheres com 32 a 37 anos $(50 \%)$, pardas $(68,75 \%)$, casadas $(37,5 \%)$, com ensino médio completo $(37,5 \%)$ com renda familiar a maioria sem empregos (56,25\%). Possuíam histórico familiar de hipertensão $(87,5 \%)$, e diabetes (50\%), eram multíparas $(43,75 \%)$ e apresentavam obesidade (75\%). O acompanhamento do pré-natal continua sendo uma das medidas importante na prevenção e no cuidado da saúde do biônimo (mãe-feto) contribuindo para a redução dos casos de mortalidade materna fetal, visto que a SHEG ainda é considerada uma importante complicação durante o período gravídico-puerperal, sendo de elevado risco de mortalidade materna e perinatal representando elevadas taxas de incidência e prevalência.
\end{abstract}

Palavras-chave: Hipertensão induzida pela gravidez; Gravidez de alto risco; Assistência pré-natal.

\begin{abstract}
Most pregnancies are uneventful, however some women have an unfavorable evolution classified as high-risk pregnancy. Among the diseases that can develop during pregnancy, the Specific Hypertensive Syndrome in Pregnancy (SHEG) stands out, considered one of the main causes of fetal maternal mortality. The aim of this study is to know the profile of pregnant women attended at Basic Health Units (UBS) in the city of Cáceres-MT in 2020, in order to identify the risk factors that influence the onset of the disease and its complications. This is a qualitative and quantitative descriptive field study, carried out with 16 pregnant women between the months of June and July 2020. For data collection, a questionnaire with closed and open questions was designed and applied. There was a higher prevalence of women aged 32 to 37 years $(50 \%)$, brown $(68.75 \%)$, married $(37.5 \%)$, with complete high school $(37.5 \%)$ with family income and mostly without jobs $(56,25 \%)$. They had a family history of hypertension $(87.5 \%)$ and diabetes $(50 \%)$, were multiparous $(43.75 \%)$ and were obese $(75 \%)$. Prenatal monitoring remains one of the important measures in the prevention and health care of the bionym (mother-fetus) contributing to the reduction of cases of fetal maternal mortality, since SHEG is still considered an important complication during the period
\end{abstract}


pregnancy and childbirth, with high risk of maternal and perinatal mortality, representing high rates of incidence and prevalence.

Keywords: Pregnancy-induced hypertension; High-risk pregnancy; Prenatal care.

\section{Resumen}

La mayoría de los embarazos transcurren sin incidentes, sin embargo, algunas mujeres tienen una evolución desfavorable clasificada como embarazo de alto riesgo. Entre las enfermedades que pueden desarrollarse durante el embarazo, se destaca el Síndrome Hipertensivo Específico en el Embarazo (SHEG), considerado una de las principales causas de mortalidad materna fetal. El objetivo de este estudio es conocer el perfil de las gestantes atendidas en las Unidades Básicas de Salud (UBS) de la ciudad de Cáceres-MT en 2020, con el fin de identificar los factores de riesgo que influyen en la aparición de la enfermedad y sus complicaciones. Se trata de un estudio de campo descriptivo cualitativo y cuantitativo, realizado con 16 gestantes entre los meses de junio y julio de 2020. Para la recolección de datos se diseñó y aplicó un cuestionario con preguntas cerradas y abiertas. Hubo una mayor prevalencia de mujeres de 32 a 37 años (50\%), morenas (68,75\%), casadas (37,5\%), con estudios secundarios completos $(37,5 \%)$ con ingresos familiares y en su mayoría sin trabajo $(56,25 \%)$. Tenían antecedentes familiares de hipertensión $(87,5 \%)$ y diabetes $(50 \%)$, eran multíparas $(43,75 \%)$ y eran obesos $(75 \%)$. El monitoreo prenatal sigue siendo una de las medidas importantes en la prevención y atención de la salud del biónimo (madre-feto) que contribuye a la reducción de los casos de mortalidad materna fetal, ya que la SHEG todavía se considera una complicación importante durante el período de embarazo y parto, con una alta riesgo de mortalidad materna y perinatal, que representa altas tasas de incidencia y prevalencia.

Palabras clave: Hipertensión inducida por el embarazo; Embarazo de alto riesgo; Cuidado prenatal.

\section{Introdução}

A maioria das gestações transcorre sem intercorrências, entretanto, algumas mulheres apresentam evolução desfavorável sendo classificadas de gravidez de risco (Oliveira \& Graciliano, 2015). Entre as doenças que podem ser desenvolvidas no período gestacional destaca-se a Síndrome Hipertensiva Especifica na Gestação (SHEG).

A SHEG é uma intercorrência obstétrica caracterizada por manifestações clinicas como a Hipertensão Arterial Sistêmica (HAS)após a vigésima semana de gestação, podendo ser associada pela presença de proteinúria, denominada como pré-eclâmpsia e nos casos mais graves a gestante pode apresentar quadro convulsivo associado aos relatados anteriormente caracterizando a eclampsia (Araújo, Santos \& Franklin, 2017; Silva et al., 2021).

No mundo, a SHEG é uma das principais causas de mortalidade materna fetal; nos países desenvolvidos essa incidência varia entre 2 a $8 \%$ das gestações, enquanto no Brasil atingi cerca de $10 \%$ das gestantes, constituindo a primeira causa de morbimortalidade materna fetal (Bacelar, Costa, Gama, Amaral \& Almeida, 2017).

O Ministério da Saúde(MS) classifica as SHEG como: Hipertensão crônica (observada antes da gravidez, ou antes da 20 semanas de gestação, ou diagnosticada pela primeira vez durante a gravidez e não se resolve até doze semanas após o parto); Pré-eclâmpsia/eclampsia hipertensão que ocorre após a 20 semanas de gestação( ou antes, em casos de doença trofoblástica gestacional ou hidrópsia fetal, acompanhada de proteinúria com desaparecimento até 12 semanas pós-parto); préeclâmpsia sobreposta à hipertensão crônica( pré eclampsia em mulheres com hipertensão crônica ou doença renal), e Hipertensão gestacional( sem proteinúria) (Brasil, 2012). Dentro das SHEG, a pré-eclâmpsia deve receber uma atenção especial que ocorre como forma isolada ou associada à hipertensão crônica sendo relacionada aos piores desfechos maternos e perinatais (Araújo et al., 2017).

Desse modo, é importante destacar os fatores de riscos que estão associados no surgimento das síndromes, tais como: obesidade, idade <16 anos, idade materna avançada, diabetes, hipertensão, nefropatias, história familiar ou pessoal de préeclâmpsia ou eclâmpsia, dietas hipoprotéicas e hipersódicas, baixa escolaridade e atividade profissional fora do domicílio, grupo sanguíneo AB, primigestas, gestações múltiplas, hidropsia fetal e neoplasia trofoblástica (Bacelar et al., 2017).

As complicações da HAS na gestação provocam, principalmente por abortamento, parto prematuro, restrição do crescimento fetal, descolamento da placenta, sofrimento fetal e afecções em órgãos vitais após o nascimento, nas situações mais graves a doença evolui para pré-eclâmpsia, eclâmpsia ou síndrome hemólise, elevação de enzimas hepáticas e baixa 
contagem de plaquetas (HELLP): tais síndromes contribuem no aumento do risco para a vida materna (Moura, Castro, Margotto \& Rugolo, 2011).

Entende-se que o diagnóstico precoce da HAS e o tratamento adequado são fundamentais para mudanças dos resultados desfavoráveis e considera-se a hipertensão na gestação quando os valores absolutos de Pressão Arterial (PA) sistólica $\geq 140 \mathrm{mmHg}$ e/ou diastólica de $\geq 90 \mathrm{mmHg}$, o diagnóstico é realizado pela medida seriada dos níveis pressóricos durante o acompanhamento pré-natal (Souza et al.,2020).

O tratamento da SHEG consiste na redução da pressão sanguínea materna e aumento do fluxo sanguíneo placentário, a hidralazina e a metildopa são as drogas mais utilizadas como anti-hipertensivos na gestação esses medicamentos agem promovendo o relaxamento do músculo liso das arteríolas periféricas e a redução da resistência vascular (Ferrão et al., 2006).

Umas das medidas de prevenção da SHEG é o acompanhamento da gestante no pré-natal, essas consultas do pré-natal devem ser realizadas pela medicina e pela enfermagem, com o objetivo de: diagnosticar ou identificar enfermidades existentes, realizar o acompanhamento da evolução da gravidez, observando as condições materna e o desenvolvimento fetal, além de diagnosticar e tratar intercorrências clínicas referentes à gravidez, as gestantes identificadas com hipertensão deverão ser referenciadas para realização do pré-natal em serviço especializado de alto risco ( Brasil, 2012). Aquelas que apresentarem quadros de pré-eclâmpsia ou eclampsia, deverão ser encaminhadas imediatamente aos serviços de urgência e emergências obstétricas (Caldas, Silva, Böing, Crepaldi, \& Custódio, 2013; Silva et al.,2021).

Em 2000, foi implantado no Brasil, o Programa de Humanização do Parto e do Nascimento (PHPN) e criado um protocolo com ações recomendadas visando a diminuição da mortalidade materna e perinatal, em 2004, o MS lançou a "Política Nacional de Atenção Integral à Saúde da Mulher" (PNAISM) que assegura a necessidade de ações voltadas a atenção à saúde a qual contribui para a redução da morbimortalidade feminina em todos os ciclos de vida, essa política estimula a participação da enfermagem nas ações de saúde da mulher especialmente no pré-natal (Costa, Vila, Rodrigues, Martins \& Pinho, 2013).

O enfermeiro tem um papel de grande importância, esse profissional acompanha a gestante desde a descoberta da gravidez e no decorrer do pré-natal, o parto, o puerpério e após-alta hospitalar, durante o acompanhamento da gestante o enfermeiro também tem o cuidado de zelar tanto pela saúde da mãe e do feto tendo a capacidade de detectar sinais de riscos e prevenir eventuais complicações quando possível (Fassarella et al.,2020).

Considerando a gravidade e a incidência da SHEG o presente estudo teve como objetivo conhecer o perfil das gestantes com SHEG atendidas nas UBS na cidade de Cáceres-Mato Grosso no ano de 2020, visando identificar os fatores de riscos que influenciam no aparecimento da doença e suas complicações.

\section{Metodologia}

\subsection{Tipo de Estudo}

Trata-se de um estudo de campo de caráter descritivo de abordagem quali-quantitativo, realizado nas UBS do município de Cáceres (MT). Para pereira et al. (2018) Os métodos qualitativos e quantitativo são importantes porque se complementam permitindo um melhor entendimento dos fenômenos em estudo.

\subsection{Local do Estudo}

O município de Cáceres pertence ao Estado de Mato Grosso e situa-se a 211 quilômetros da capital (Cuiabá) e possui uma população estimada com 94.861 pessoas (IBGE, 2020). Atualmente a cidade de Cáceres conta com 12 UBS nas urbanas, sendo as seguintes: Cohab Nova, Jardim Guanabara, Jardim Paraiso, Jardim Padre Paulo, Santos Dumont, Marajoara, Rodeio, Santa Isabel, Vila Irene, Vila Real, CAIC e Vitoria Régia. 


\subsection{Amostra}

A amostra foi composta por 16 gestantes adultas que estavam realizando a consulta de pré-natal nas UBS. A escolha da amostra da pesquisa foi feita por conveniência, isto é, participaram da pesquisa somente as mulheres que aceitaram colaborar com a realização deste estudo quando convidados (Oliveira, 2001).

Das 170 mulheres que estavam realizando o pré-natal nas UBS, apenas 16 atendiam os critérios de inclusão, e 154 gestantes não atendiam aos critérios de inclusão: 108 tinham o pré-natal de baixo risco; 39 eram gestantes adolescentes e 7 (sete) foram encaminhadas ao pré-natal de risco para acompanhamento da equipe multidisciplinar e no momento não havia retornado para dar continuidade na UBS de origem.

As 16 (dezesseis) gestantes eram cadastradas nas seguintes UBS: CAIC (três gestantes), Guanabara (três gestantes), Jardim Paraiso (cinco gestantes), Santos Dumont (uma gestante), Vila Irene (três gestantes), Vitória Régia (uma gestante). A escolha das unidades ocorreu através do sorteio, realizado da seguinte forma: os nomes das UBS foram escritos em um papel e colocados em um recipiente de vidro e sorteado 50\% das 12 UBS, sendo o total de seis unidades. Definiu como critérios de inclusão: ter idade 19 a 43 anos de idade, possuir diagnóstico médico de hipertensão, estar realizando acompanhamento do pré-natal nas UBS da zona urbana, independentemente da Idade Gestacional (IG), e os critérios de exclusão foram: ser gestantes adolescentes, mulheres com pré-natal de baixo risco, gestantes procedentes das UBS da zona rural.

\subsection{Coleta de dados}

Para a coleta de dados foi elaborado e aplicado um questionário com perguntas fechadas e abertas abordando questões sobre as características sociodemográficas: idade, raça/cor, escolaridade, estado civil e renda familiar, as variáveis sobre histórico familiar( hipertensão e diabetes), antecedentes obstétricos( número de gestação, tipo de parto, préeclâmpsia/eclampsia), sinais clínicos da hipertensão( cefaleia, visão embaçada, aceleração do ritmo cardíaco, aumento da PA, dor abdominal, edema em membros superiores e inferiores) e gestação atual.

A pesquisas ocorreu nos período de junho a julho de 2020, em seis UBS uma vez por semana nos dias de atendimento da consulta de pré-natal estabelecido por cada unidade( terça, quarta e quinta-feira ), com os profissionais de saúde (médico ou enfermeiro) ou por agendamento do local(domicilio), data e dia de preferido pela gestante, devido o momento da pandemia do Coronavírus(SARS-COV-2) COVID 19, para a segurança da saúde da gestante e do pesquisador foram tomadas todas as medidas preventivas para evitar a contaminação e a disseminação do vírus como o uso de máscara, higienização das mão com álcool em gel a 70\% e o distanciamento de 2 metros.

\subsection{Analises dos dados}

Para a análise dos resultados, os dados coletados foram organizados em uma planilha eletrônica no programa Microsoft Office Excel@ 2013. Após a tabulação, adotou-se tratamento estatístico, emprego de frequências absoluta e percentuais.

\subsection{Aspectos éticos}

O projeto de pesquisa foi submetido à avaliação do Comitê de Ética em Pesquisa da Universidade do Estado de Mato Grosso CEP/UNEMAT, para atender a resolução 466/2012 e a norma operacional 001/2013 do CNS (Conselho Nacional de Saúde) aprovado em junho de 2020 sob o No parecer 4.121.359 e do Certificado de Apresentação de Apreciação Ética (CAAE): 33487520.9.0000.5166. 
Para manter o anonimato as gestantes foram identificadas pela letra $(\mathrm{G})$ de gestante e codificada pelos algarismos $(1,2 \ldots 16)$. As participantes foram orientadas quantos aos riscos e benefícios do estudo. Após a aceitação, leitura e assinatura do Termo de Consentimento Livre e Esclarecido (TCLE) foi iniciada a pesquisa.

\section{Resultados e Discussão}

Participaram da pesquisa 16 gestantes assistida em 6 UBS, na faixa etária entre 20 e 43 anos, com a idade média de 30,4 anos, a maior prevalência da SHEG ocorreu em mulheres com idade entre 32 e 37 anos 8 (50\%); na cor pardas 11(68,75\%); casadas 6(37,5\%); concluíram o ensino médio 6(37,5\%) seguido do ensino superior 5(31,25\%) sendo a renda familiar desempregadas $9(56,25 \%)$. Tabela 1 .

Tabela 1. Distribuição das gestantes com síndrome hipertensiva, segundo as características sociodemográficas, Cáceres-MT, 2020 .

\begin{tabular}{|c|c|c|}
\hline Características sociodemográficas & $\begin{array}{l}\text { Frequência } \\
\text { Absoluta(n) }\end{array}$ & $\begin{array}{c}\text { Frequência } \\
\text { Relativa (\%) }\end{array}$ \\
\hline \multicolumn{3}{|l|}{ Faixa etária } \\
\hline $20-25$ anos & 5 & 31,25 \\
\hline $26-31$ anos & 2 & 12,5 \\
\hline $32-37$ anos & 8 & 50 \\
\hline $38-43$ anos & 1 & 6,25 \\
\hline Média & 30,4 & \\
\hline \multicolumn{3}{|l|}{ Raça/cor } \\
\hline Branca & 4 & 25 \\
\hline Negra & 1 & 6,25 \\
\hline Parda & 11 & 68,75 \\
\hline \multicolumn{3}{|l|}{ Escolaridade } \\
\hline Ensino Fundamental Incompleto & 4 & 25 \\
\hline Ensino Médio completo & 6 & 37,5 \\
\hline Ensino Superior completo & 5 & 31,25 \\
\hline Ensino Superior Incompleto & 1 & 6,25 \\
\hline \multicolumn{3}{|l|}{ Estado civil } \\
\hline Casada & 6 & 37,5 \\
\hline Solteira & 5 & 31,25 \\
\hline União estável & 5 & 31,25 \\
\hline \multicolumn{3}{|l|}{ Renda familiar } \\
\hline 1 a 2 salários & 7 & 43,75 \\
\hline Desempregada & 9 & 56,25 \\
\hline Total & 16 & $100 \%$ \\
\hline
\end{tabular}

Fonte: Autores.

Quanto aos antecedentes obstétricos houve prevalência de mulheres multíparas 7(43,75) seguido de primigestas 5(31,25); sendo a via de parto predominante parto cesáreo 8(50\%); tiveram pré-eclâmpsia/ eclampsia 3(18,75\%), e hipertensão na gestação anterior 7(43,75\%), possuíam histórico familiar para hipertensão 14(87,5\%) e diabetes 8(50\%) (Tabela 2). 
Tabela 2. Distribuição das gestantes com síndrome hipertensiva, segundo os antecedentes obstétricos e histórico familiar Cáceres-MT, 2020.

\begin{tabular}{|c|c|c|}
\hline Antecedentes obstétricos & $\begin{array}{l}\text { Frequência } \\
\text { Absoluta(n) }\end{array}$ & $\begin{array}{c}\text { Frequência } \\
\text { Relativa }(\%)\end{array}$ \\
\hline \multicolumn{3}{|l|}{ Número de gestação } \\
\hline Primigestas & 5 & 31,25 \\
\hline Secundigestas & 4 & 25 \\
\hline Multíparas & 7 & 43,75 \\
\hline \multicolumn{3}{|l|}{ Tipo de parto } \\
\hline Cesáreo & 8 & 50 \\
\hline Vaginal & 4 & 25 \\
\hline Nenhum parto & 4 & 25 \\
\hline \multicolumn{3}{|l|}{ Pré-eclâmpsia e eclampsia } \\
\hline Não & 13 & 81,25 \\
\hline Sim & 3 & 18,75 \\
\hline \multicolumn{3}{|c|}{ Hipertensão na gestação anterior } \\
\hline Não & 9 & 56,25 \\
\hline Sim & 7 & 43,75 \\
\hline \multicolumn{3}{|c|}{ Histórico familiar de hipertensão } \\
\hline Não & 2 & 12,5 \\
\hline Sim & 14 & 87,5 \\
\hline \multicolumn{3}{|c|}{ Histórico familiar de diabetes mellitus } \\
\hline Não & 8 & 50 \\
\hline Sim & 8 & 50 \\
\hline Total & 16 & $100 \%$ \\
\hline
\end{tabular}

Fonte: Autores.

Em relação a gestação atual, identificou-se a maioria das mulheres tinham a IG das gestantes entre 20 e 22 semanas $7(43,75 \%)$; tiveram diagnóstico de hipertensão no segundo trimestre $9(56,25 \%)$ e no terceiro trimestre $5(31,25 \%)$; não planejaram a gravidez $11(68,75 \%)$; realizaram entre 4 a 6 consultas 10 (62,5\%) e os exames pré-natal $15(93,75 \%)$, estavam em uso de hipertensivos 14 (87,5\%); apresentavam obesidade $12(75 \%)$ e sedentarismo 10 (62,5\%) (Tabela 3). 
Tabela 3. Distribuição das gestantes com síndrome hipertensiva, segundo a gestação atual, Cáceres, MT, 2020.

\begin{tabular}{|c|c|c|}
\hline $\begin{array}{c}\text { Gestação } \\
\text { Atual }\end{array}$ & $\begin{array}{l}\text { Frequência } \\
\text { Absoluta(n) }\end{array}$ & $\begin{array}{c}\text { Frequência } \\
\text { Relativa (\%) }\end{array}$ \\
\hline \multicolumn{3}{|l|}{ Idade Gestacional } \\
\hline 20 a 22 semanas & 7 & 43,75 \\
\hline 24 a 29 semanas & 6 & 37,5 \\
\hline 32 a 40 semanas & 3 & 18,75 \\
\hline \multicolumn{3}{|c|}{ Diagnóstico da hipertensão } \\
\hline 1 trimestre & 2 & 12,5 \\
\hline 2 trimestres & 9 & 56,25 \\
\hline 3 trimestres & 5 & 31,25 \\
\hline \multicolumn{3}{|l|}{ Gravidez planejada } \\
\hline Não & 11 & 68,75 \\
\hline Sim & 5 & 31,25 \\
\hline \multicolumn{3}{|l|}{ Número de consultas } \\
\hline 1 a 3 & 6 & 37,5 \\
\hline 4 a 6 & 10 & 62,5 \\
\hline \multicolumn{3}{|c|}{ Exames de pré-natal realizados } \\
\hline Não & 1 & 6,25 \\
\hline Sim & 15 & 93,75 \\
\hline \multicolumn{3}{|l|}{ Uso de hipertensivo } \\
\hline Não & 2 & 12,5 \\
\hline Sim & 14 & 87,5 \\
\hline \multicolumn{3}{|l|}{ Peso pré-gestacional } \\
\hline Obesidade & 12 & 75 \\
\hline Peso adequado & 1 & 6,25 \\
\hline Sobrepeso & 3 & 18,75 \\
\hline \multicolumn{3}{|l|}{ Tipo de exercícios } \\
\hline Caminhada & 5 & 31,25 \\
\hline Exercícios leves & 1 & 6,25 \\
\hline Sedentárias & 10 & 62,5 \\
\hline Total & 16 & $100 \%$ \\
\hline
\end{tabular}

Fonte: Autores.

Quanto aos sinais e sintomas a maioria das gestantes apresentaram sintomas de cefaleia 12 (75\%); visão embaçada 11 (68\%); aceleração do ritmo cardíaco 8 (50\%); aumento da pressão arterial 15 (93,75\%); dor abdominal 4(25\%), presença de edema em membros superiores e inferiores 11 (68,75\%). (Tabela4). 
Tabela 4. Distribuição das gestantes com síndrome hipertensiva, segundo os sinais e sintomas, Cáceres, MT, 2020.

\begin{tabular}{|c|c|c|}
\hline Sinais e sintomas & $\begin{array}{l}\text { Frequência Absoluta } \\
\text { (n) }\end{array}$ & $\begin{array}{c}\text { Frequência } \\
\text { Relativa (\%) }\end{array}$ \\
\hline \multicolumn{3}{|l|}{ Cefaleia } \\
\hline Não & 4 & 25 \\
\hline Sim & 12 & 75 \\
\hline \multicolumn{3}{|l|}{ Visão embasada } \\
\hline Não & 5 & 31,25 \\
\hline Sim & 11 & 68,75 \\
\hline \multicolumn{3}{|c|}{ Aceleração do ritmo cardíaco } \\
\hline Não & 8 & 50 \\
\hline Sim & 8 & 50 \\
\hline \multicolumn{3}{|l|}{ PA $>140 / 90 \mathrm{mmhg}$} \\
\hline Não & 1 & 6,25 \\
\hline Sim & 15 & 93,75 \\
\hline \multicolumn{3}{|l|}{ Dor abdominal } \\
\hline Não & 12 & 75 \\
\hline Sim & 4 & 25 \\
\hline \multicolumn{3}{|c|}{$\begin{array}{c}\text { Edema em membros superiores e } \\
\text { inferiores }\end{array}$} \\
\hline Não & 5 & 31,25 \\
\hline Sim & 11 & 68,75 \\
\hline Total & 16 & $100 \%$ \\
\hline
\end{tabular}

Fonte: Autores.

O estudo demonstrou que a idade materna avançada, cor, renda familiar, histórico familiar para hipertensão e diabetes, antecedentes obstétricos e a obesidade são as características comum entre as gestantes que apresentaram SHEG no município de Cáceres. Ressalta-se que estas características são apontadas na literatura como fatores de riscos para o surgimento da SHEG (Souza et al., 2020).

Neste estudo, identifica-se maior prevalência da SHEG em gestantes com idade mais avançada comparada a outros estudos. (Oliveira \& Graciliano, 2015; Moura et al., 2011). Já que 50\% das gestantes tinham entre 32 a 37 anos. Tais números apontaram a gestação tardia. Concordando com os dados do MS, a idade igual ou maior de 35 anos é apontada como fator de risco gestacional, sendo considerado importante fator de risco para a morbimortalidade materno-fetal, reforçando os achados da presente pesquisa (Brasil, 2012).

Segundo Bacelar et al. (2017) e Brasil (2012), a idade materna avançada é citada como fator de risco para o surgimento de complicações durante o ciclo gravídico, dentre elas a pré-eclâmpsia, que pode ocorrer tanto em mulheres jovens quanto nas com idade avançada, no entanto, a incidência da SHEG é mais frequente em adolescentes menores de 16 anos, que estejam apresentando estado nutricional deficiente e ausência de cuidados pré-natais.

Em relação a cor, o maior percentual da SHEG ocorreu em mulheres pardas $(68,75 \%)$. Concordando com uma pesquisa realizada em uma maternidade do Pará por Guerreiro, Borges, Nunes, Silva e Maciel (2014) sobre a mortalidade materna da SHEG observaram que $(60,6 \%)$ de gestantes eram pardas. Outro estudo realizado no Maranhão por Alves et al. (2014), sobre o perfil das pacientes com SHEG observaram (50\%) eram pardas. No entanto Souza et al. (2020) estudando a epidemiologia da hipertensão em gestantes observaram que $(61,4 \%)$ eram brancas, essas características podem estar relacionadas aos perfis da gestante e do local onde a pesquisa foi realizada.

Neste estudo, observa-se o aumento da escolaridade entre as gestantes a maioria concluíram ensino médio completo $(37,5 \%)$ seguido do ensino superior completo $(31,25 \%)$. Semelhante ao estudo realizado por Souza et al. (2020), sobre a epidemiologia da hipertensão arterial em gestantes identificaram que $(21,1 \%)$ completaram o ensino médio completo, seguido 
do ensino superior completo (37,7\%). A elevação do nível de escolaridade materna reduz as taxas de morbimortalidade perinatal e das complicações gestacionais (Peixoto et al., 2012).

Quanto a renda familiar a maior parcela das gestantes estava sem empregos (56,25\%). A literatura aponta a baixa renda familiar fator de risco na gestação, haja vista que a mesma tem levado algumas mulheres à gestação de risco e associadas ao estresse e piores condições nutricionais, o estresse está relacionado ao desemprego bem como a difícil inserção no mercado de trabalho ambos contribuem para distúrbios hipertensivos na gestação (Moura, Oliveira, Damasceno \& Pereira, 2010).

No que refere a situação conjugal a maioria eram casadas (37,5\%), sendo está variável não considerado fator de risco nesse estudo. De acordo com Souza et al. (2020), algumas mulheres preferem engravidar dentro de um relacionamento mais consolidado, haja vista que a estabilidade colabora para a evolução da gestação, do estado emocional e financeiro, porém o fato das mulheres serem casadas não impedem que mesmas desenvolver a SHEG na gestação.

Para Souza, Queiroz, Queiroz, Ribeiro e Fonseca (2013) as mulheres que possuem apoio do parceiro durante a gravidez vivenciam este momento com mais tranquilidade, mesmo se tratando de uma gestação de risco contribuindo positivamente para a boa evolução da gestação e no seguimento das orientações.

Quanto ao histórico familiar, observa-se a prevalência da hipertensão (87,5\%) e diabetes (50\%). Concordando com estudo realizado na cidade de São Paulo por Souza et al. (2020), sobre a epidemiologia da hipertensão em gestantes identificaram histórico familiar para hipertensão $(78,1 \%)$ e diabetes $(50 \%)$. Outro estudo realizado no Instituto da mulher em Francisco Beltrão no estado do Paraná por Costa, Cura, Perondi, França e Bortoloti (2016), observaram histórico familiar para hipertensão $(63,9 \%)$ e para diabetes $(34,4 \%)$ em gestante de alto risco.

A presença de um ou mais fator de risco aumenta as chances de complicação gestacional, como distúrbios hipertensivos e o nascimento de pré-termos. (Moura et al., 2010; Rodrigues, Dantas, Pereira, Silveira \& Rodrigues, 2017).

Referente aos antecedentes obstétricos, observa-se a prevalência de mulheres multíparas (43,75\%) e primigestas (31,25\%) via de parto cesáreo (50\%). Estudo realizado no Maranhão por Alves et al. (2014), sobre o perfil das pacientes com SHEG internadas em uma Unidade de Terapia Intensiva (UTI), observaram que a maioria $(51,7 \%)$ eram primigestas, seguidas de multíparas (39,7\%) apresentando complicações de eclampsia, hemorragia, edema agudo de pulmão, Hellp síndrome.

De acordo com Brito et al. (2015), a SHEG tem sido associada com o aumento do número de parto cesáreo, a justificativa é devido a síndrome apresentar complicações materno fetal, que na maioria dos casos só resolvem com a interrupção da gravidez. No entanto MS, recomenda o parto vaginal para a gestação de risco, salienta ainda que a gravidez de risco não é sinônimo de parto cirúrgico, em algumas situações é possível a indução do parto por via vaginal ou aguardar seu início espontâneo (Brasil, 2012).

No que refere a idade gestacional é comum que o diagnóstico da hipertensão ocorra a partir da $20^{\mathrm{a}}$ semana fato este que caracteriza a SHEG (Souza et al.,2020). Fato este observado na presente pesquisa (43,75\%) das gestantes tinham entre $20 \mathrm{e}$ 22 semanas, sendo diagnosticadas no segundo $(56,25 \%)$ e no terceiro trimestre de gestação $(31,25 \%)$. Importante salientar que $(43,75 \%)$ responderam tiveram hipertensão na gestação anterior, ou seja, a maioria das gestantes já eram hipertensas.

A SHEG pode se apresentar como hipertensão crônica observada antes da gestação ou até a $20^{\text {a }}$ semana gestacional, sendo a pré eclampsia definida pela presença da hipertensão após a 20 semana de gestação acompanhada de proteinúria, desaparecendo após o parto (Brasil, 2012; Brito et al., 2015).

Quanto ao planejamento da gestação, a maioria das mulheres declararam não ter planejado a gravidez $(68,75 \%)$ realizaram entre 4 e 6 consultas (62,5\%) e os exames de pré-natal (93,75\%). Para Ferrão et al. (2006), a gravidez quando planejada possibilita o desenvolvimento mais tranquilo, mesmo nos casos da gestação de alto risco. Os dados desse estudo revelam que embora as mulheres não planejaram a gravidez, no entanto, observa-se uma boa adesão na realização dos exames e das consultas de pré-natal. 
Segundo o MS é recomendado que a gestante realize no mínimo 6 consultas de pré-natal, sendo mensais até a $28^{a}$ semana, quinzenais entre 28 e 36 semanas e semanais até que venha a termo, no entanto, as gestantes de risco devem realizar um número mais elevado, visto que estas possuem uma complicação gravídica (Brasil, 2012).

Com relação ao peso pré-gestacional, após a análises observa-se que a grande maioria das mulheres apresentavam obesidade (75\%), isto significativa que as mulheres estavam acima do peso antes de engravidarem. Nogueira et al. (2020) concluíram em seu estudo que o estado nutricional no início da gestação e durante o período gestacional tem uma associação positiva com as SHEG, sendo este um fator risco.

Na gestação a obesidade está associada ao aparecimento de diabetes mellitus gestacional (DMG), distúrbios hipertensivos, macrossomia, pré-eclâmpsia óbito fetal, parto prematuro e mortalidade perinatal (Lacerda \& Moreira, 2011; Oliveira \& Graciliano, 2015). Concordando com a literatura, o presente estudo demonstrou que a obesidade a hipertensão foram características associados com o aparecimento da SHEG.

Nesse sentindo acompanhamento do pré-natal precoce torna-se uma estratégia importante com orientações quanto ao ganho e o controle do ganho peso no período gestacional e sobre as práticas de atividades físicas na gravidez.

Nascimento, Godoy, Surita e Silva (2014), traz em seu estudo que na ausência de contraindicação para a realização de atividade física na gestação, todas as gestantes devem ser estimuladas a manter ou adotar um estilo de vida ativo, sendo indicados os exercício físicos de intensidade leve a moderada considerados seguro tanto para a mãe quanto ao feto, porém as gestantes com diagnóstico ou suspeita de pré-eclâmpsia devem evita-los, visto que o mesmo aumenta a PA e reduz o fluxo uteroplacentário.

Moura et al. (2010) citam em seu estudo que todas as gestantes devem ter uma alimentação saudável, balanceada e diversificada, sendo recomendado seguimento da pirâmide alimentar, a dieta da gestante hipertensa segue as recomendações da gestação de baixo risco, evitando o excesso de gorduras e açúcares.

No entanto, nem todas as gestantes conseguem manter ou aderir uma alimentação saudável, visto que isto depende do fator econômico, quanto mais elevada a renda maior a inclusão de alimentos saudáveis, quanto menor a renda, menos inclusão desses alimentos (Ferreira \& Magalhães, 2011).

Quanto aos sinais e sintomas, a maioria das gestantes apresentaram todos os sintomas investigados, no entanto os mais evidentes foram cefaleia (75\%) aumento da PA $(93,75 \%)$ e edema nos membros superiores e inferiores $(68,75 \%)$. Um estudo realizado na cidade de Fortaleza no Ceará por Lacerda e Moreira (2011), observaram que os sintomas apresentados pelas as gestantes com pré-eclâmpsia foram cefaleia (29\%), edema de membros superiores e inferiores (64 \%) tontura (20,4\%) e dores em baixo ventre $(18,1 \%)$. Sendo importante que os profissionais de saúde estejam atentos as queixas e aos relatos apresentados pelas gestantes.

Segundo o MS, na ausência de proteinúria a suspeita de pré-eclâmpsia/eclampsia se fortalece quando o aumento da PA aparece acompanhada por cefaleia, distúrbios visuais, dor abdominal, plaquetopenia e aumento de enzimas hepáticas (Brasil, 2012).

A pré-eclâmpsia, caracterizada pela elevação da PA, alguns sinais são indicativos desta condição como a presença de edema na face, mãos, membros inferiores ou edema generalizado (Moura et al., 2010). Salientamos que os sinais clínicos da hipertensão devem receber uma atenção especial pelos profissionais de saúde e devem ser diagnostico e monitorados o quanto antes para um desfecho favorável da gestante.

Quanto ao tratamento medicamentoso as gestantes estavam em uso de hipertensivos para o controle da PA. De acordo com o manual técnico da gestação de alto risco do MS, a metildopa é a droga mais utilizada para o tratamento da SHEG, no entanto, podem causar sonolência, em caso de resistência à terapia avaliar a possibilidade de retenção de sal e nesse caso o uso de diurético pode ser associado a uma alimentação saudável (Brasil, 2012). 
Para Abrahão et al. (2020), o enfermeiro possuir competência e formação para aplicar seus conhecimentos técnico e científico na assistência, sendo a Sistematização da Assistência em Enfermagem (SAE) uma atribuição exclusiva do enfermeiro no planejamento dos cuidados a gestante, diferenciando-o dos demais profissionais da equipe multidisciplinar.

\section{Considerações Finais}

Nesse estudo, por meio da análise das características sociodemográficas, tornou-se possível conhecer o perfil das mulheres com síndromes hipertensiva especifica da gestação atendidas nas Unidades Básicas de Saúde em Cáceres Mato Grosso, Brasil e a identificação das características comum do grupo, que são semelhantes aos fatores de riscos, que contribuem para o surgimento da síndrome e suas principais complicações apontadas em estudos científicos.

A idade materna avançada, renda familiar, histórico familiar, antecedentes obstétricos e a obesidade, são aspectos que podem influenciar de forma negativa na gestação contribuindo para um resultado desfavorável. A prevalência da obesidade e do sedentarismo entre as gestantes torna-se preocupante visto que se trata de fatores modificáveis, tem sido comumente associada com o surgimento de complicações durante o período gestacional, uma delas são as SHEG, portanto, merece uma atenção pelos profissionais de saúde em especial o enfermeiro.

Diante disso, sugere que os profissionais realizem educação em saúde com orientações sobre a alimentação saudável, adesão as práticas de atividades físicas, ressaltando seus benefícios como controle do ganho de peso e também a importância das consultas do pré-natal.

Nesse sentindo, essa pesquisa contribuirá aos profissionais de saúde da atenção básica a partir do conhecimento do perfil das gestantes os mesmos poderá identificar uma gestação de risco, possibilitando no planejamento de estratégia de saúde individual para cada mulher. Sabe-se que o pré-natal, continua sendo uma das medidas de prevenção no cuidado da saúde do biônimo mãe-feto, o mesmo contribuir para a redução dos casos de mortalidade materna fetal, visto que a SHEG ainda é considerada um importante complicações durante o período gravídico-puerperal, sendo de elevado risco de mortalidade materna e perinatal representando elevadas taxas de incidência e prevalência.

Este estudo apresentou algumas limitações dentre elas o enfrentamento da pandemia da covid 19, sendo observado no baixo índice de participação de gestantes, que não se sentiam segura por medo de se contaminar com o vírus, mesmo sendo seguido todos os protocolos de segurança recomendo pela Organização Mundial de Saúde (OMS).

Como recomendação sugere-se a continuidade de estudos futuros que abordem a temática mais aprofundada, sendo importante a inclusão também das gestantes adolescentes afim de compreender melhor o surgimento das síndromes hipertensivas em todas as faixas etárias.

\section{Referências}

Abrahão, A.C. M., Santos, R. F. S., Viana, S. R. G., Viana, S. M. \& Costa, C. S. C. (2020). Atuação do enfermeiro a pacientes portadoras de Síndrome Hipertensiva Especifica da Gestação. Rev Cient Esc Estadual Saúde Pública Goiás "Candido Santiago", 6(1): 51-63.

Alves, A. A. G., Neto, J. A. F., Sauaia, B. A., Silva, N. D. S., Chein, M. B. C., Souza, R. M. L., Padilha, D. J., Cantadori, A. S. A. \& Brito, L. M. O. (2014). Perfil clínico de pacientes com Doença Hipertensiva Específica da Gestação em uma UTI geral adulto do estado do Maranhão. Rev Pesq. Saúde, 15(1): 223229.

Araújo, I. F. M., Santos, P. A., Santos, P. A. \& Franklin, T. A. (2017). Síndromes hipertensivas e fatores de risco associados à gestação. Rev enferm UFPE on line., Recife, 11(10):4254-62. 10.5205/reuol.10712-95194-3-SM.1110sup201731

Bacelar, E. B., Costa, C. O. M., Gama, S. G. N., Amaral, M. T. R. \& Almeida, A. H. V. (2017). Fatores associados à Síndrome Hipertensiva Específica da Gestação em Puérperas adolescentes e adultas jovens da Região Nordeste do Brasil: análise Múltipla em modelos hierárquicos. Rev. Bras. Saúde Matern. Infant., Recife, 17 (4): 683-691.

Brasil. (2012). Ministério da Saúde. Secretaria de Atenção à Saúde. Departamento de ações Programáticas Estratégias.Gestação de alto risco: manual técnico. Ministério da Saúde. (5a ed.). 302 p. - (Série A. Normas e Manuais Técnicos). 
Brito, K. K. G., Moura, J. R. P., Souza, M. J., Brito, J. V., Oliveira, S. H. S. \& Oliveira, M. J. G. (2015). Prevalência das síndromes hipertensivas específicas da gestação (SHEG). J. res.: fundam. care. Online, 7(3):2717-2725. 10.9789/2175-5361. 2015.v7i3.2717-2725

Caldas, D. B., Silva, A. L. R. D., Böing, E., Crepaldi, M. A. \& Custódio, Z. A. O. (2013). Atendimento psicológico no pré-natal de alto-risco: a construção de um serviço. Psicologia Hospitalar, 11(1), 66-87.

Costa, C. S. C., Vila, V. S. C., Rodrigues, F. M., Martins, C. A. \& Pinho, L. M. O. (2013). Características do atendimento pré-natal na Rede Básica de Saúde. Rev. Eletr. Enf. 15(2):516-22.

Costa, L. D., Cura, C. C. S., Perondi, A. R., França, V. F. \& Bortoloti, D. S. (2016). Perfil Epidemiológico de Gestantes de Alto Risco. Cogitare Enferm, 21(2): 01-08.

Fassarella, B. P. A., Almeida, G., Teles, D. A., Ortiz, L. D. S.., Silva, I. S. D., Neves, K. do C., Costa, P. A. F. D. S., Ribeiro, W. A. \& Evangelista, D. da S. (2020). Nursing care aimed at pregnant women with hypertensive disease specific to pregnancy. Research, Society and Development, 9(9), e343996768. https://doi.org/10.33448/rsd-v9i9.6768

Ferrão, M. H. L., Pereira, A. C. L., Gersgorin, H. C. T. S., Paula, T. A. A., Corrêa, R. R. M. \& Castro E. C. C. (2006). Efetividade do tratamento de gestantes hipertensas. Rev Assoc Med Bras,52(6): 390-4.

Ferreira, V. A. \& Magalhães, R. (2011). Obesidade entre os pobres no Brasil: a vulnerabilidade feminina. Ciênc. Saúde Coletiva, $16(4)$ : $2279-2287$.

Guerreiro, D. D., Borges, W. D., Nunes, H. H. M., Silva, S. C. S. \& Maciel, J. P. (2014). Mortalidade materna relacionada à Doença Hipertensiva Especifica da Gestação (DHEG) em uma maternidade no Pará. Rev Enferm UFSM. 4(4):825-834.

IBGE. (2020) Instituto Brasileiro de Geografia e Estatística (IBGE). Brasil, Mato Grosso. Número da população de Cáceres estimada em 2020.

Lacerda, I. C. \& Moreira, T. M. M. (2011). Características obstétricas de mulheres atendidas por pré-eclâmpsia e eclampsia. Maringá, 33(1): 71-76-2011. 10.4025/actascihealthsci. v33i1.7711

Lima, I. M. A., Filho, C. C. S., Tavares, V. S., Espíndola, M. M. M., Nascimento, M. A. R. \& Nunes, G. F. O. (2015). Gestantes de Alto Risco: representações sociais do planejamento da gravidez, parto e família. Rev enferm UFPE on line, Recife, 9(12):1255-63. 10.5205/r euol.8127-71183-1-SM.0912201526

Moura, E. R. F., Oliveira C. G. S., Damasceno, A. K. C. \& Pereira, M. M. Q. (2010). Fatores de riscos para síndrome hipertensiva especifica da gestação entre mulheres hospitalizadas com pré-eclâmpsia. Cogitare Enferm. 15(2):250-5.

Moura, M. D. R., Castro, M. P., Margotto, P. R. \& Rugolo, L. M. S. S. (2011). Hipertensão Arterial na Gestação - importância do seguimento materno no desfecho neonatal. Com. Ciências Saúde, 22 (1):113-120.

Nascimento, S. L., Godoy, A. C., Surita, F. G. \& Silva, J. L. P. (2014). Recomendações para a prática de exercício físico na gravidez: uma revisão crítica da literatura. Rev Bras Ginecol Obstet, 36(9):423-3.

Nogueira, M.DA., Santos, C.C., Lima, A.M. et al. Associação entre estado nutricional, diabetes gestacional e doenças hipertensivas em gestantes de risco. Braz. J. of Develop., 6(2), 8005-8018.

Oliveira, A. C. M. \& Graciliano, N. G. (2015). Síndrome hipertensiva da gravidez e diabetes mellitus gestacional em maternidade pública de uma capital do Nordeste brasileiro, 2013: prevalência e fatores associados. Revista Epidemiologia e Serviços de Saúde, 24(3): 441-451.

Oliveira, M. F. (2011). Metodologia cientifica: um manual para a realização de pesquisa em administração. UFG. https://files.cercomp.ufg.b r/weby/up/567/o/Manual_de_metodologia_cientifica_-_Prof_Maxwell.pdf

Oliveira, T.M.V. Amostra não probabilística: adequação de situações para uso e limitações de amostra por conveniência, julgamento e Quotas. Administração on line pratica Pesquisa Ensino.

Peixoto, C. R., Lima, T. M., Costa, C. C., Freitas, L.V., Oliveira, A. S. \& Damasceno, C. K. A. (2012). Perfil das Gestantes Atendidas no Serviço de Pré-natal das Unidades Básicas de Saúde de Fortaleza -CE. remE-Rev. Min. Enferm.; 16(2): 171-177.

Pereira, A. S., Shitsuka, D. M., Parreira, F. J. \& Shitsuka, R. (2018). Metodologia da pesquisa científica. UFSM. https://repositorio.ufsm. br/bitstream/handle/1/15824/Lic_Computacao_Metodologia- Pesquisa-Cientifica.pdf?sequence=1

Rodrigues, A. R. M., Dantas, S. L. C., Pereira, A. M. M., Silveira, M. A. M. \& Rodrigues, D. P. (2017). Gravidez de alto risco: análise dos determinantes de saúde. Sanare, 16(1): 23-28.

Silva, E. C., Silva, N. C. D. L., Silva, A. E. G., Campos, R. L. O., Santana, M. R., Café, L.A., Almeida, P. M. O., Oliveira, S. M. Gomes, A. S. \& Silva, A. T. C. S. G. (2021). Atuação do enfermeiro na prevenção das síndromes hipertensiva na gestação no âmbito da atenção básica. Revista Eletrônica Acervo Saúde,13(2): 1-7. https://doi.org/10.25248/REAS.e6448.2021

Silva, J. R., Oliveira, M. B. T., Santos, F. D. R. P., Neto, M. S., Ferreira, A. G. N.\& Santos, F. S. (2018). Indicadores da Qualidade da Assistência Pré-Natal de Alto Risco em uma Maternidade Pública. Revista brasileira de ciências da saúde, 22(2):109-116. 10.4034/RBCS.2018.22.02.03

Souza, M. G., Lopes, R. G. C. L., Rocha, M. L. T. L. F., Lippi, U. G., Costa, E. S. \& Santos, C. M. P. (2020). Epidemiologia da hipertensão arterial em gestantes. einstein.18:1-7. 10.31744/einstein_journal/2020AO4682

Souza, N. A., Queiroz, L. L. C., Queiroz, R. C. C. S., Ribeiro, T. S. F. \& Fonseca, M. S. S. (2013). Perfil Epidemiológico das Gestantes Atendidas na Consulta de Pré-natal de uma Unidade Básica de Saúde em São Luís- Ma. Rev. Ciênc. Saúde, 15(1): 28-38. 